\title{
An Efficient Medium Access Control Strategy for High-Speed WDM Multiaccess Networks
}

\author{
Jan H. Laarhuis and A. M. J. Koonen
}

\begin{abstract}
The medium access control strategy proposed accounts for the limited tunability of present-day lasers and filters and yet, it supports a large total number of wavelengths in the network. Full interconnectivity, contention-free access and a high value of concurrency are achieved by dividing the network into disjunct subnetworks on a wavelength basis and by reconfiguring these subnetworks on a time basis. Each subnetwork allows for simplified access to be implemented with fast tunable transceivers each accessing only a moderate number of wavelengths. A performance analysis shows that this concept is most efficient when applied to a high-level broad-band interconnection MAN.
\end{abstract}

\section{INTRODUCTION}

$\mathbf{I}^{1}$ $T$ is generally recognized that WDM is one of the most attractive techniques to create multichannel networks. The different wavelength channels are mutually independent and are therefore transparent for data format, data rate and modulation format. The optical star topology is a quite suitable topology for such networks because of its inherent broadcast property and its passive character. The maximum throughput of these networks is limited to a few terabit-per-second (Tb/s) when employing direct detection and photonic amplification. However, technological constraints limit the wavelength tuning range and the tuning speed of present-day transmitters and receivers. Therefore, in the direct detection networks proposed up to now, the obtainable bandwidth decreases to a few hundred gigabits-per-second maximum [1].

In this paper a medium access control strategy and a supporting physical layer design are proposed, which maximize the network throughput at given constraints on transceiver tunability, and which maintain the transparency of the network. In Section II the main requirements of multichannel networks are described. Section III describes the physical layer architecture and the medium access control strategy. In Section IV the performance of the system is evaluated. Conclusions are given in Section $\mathrm{V}$.

\section{MUltichannel Networks}

Consider a $(N, M)$ optical star multichannel network (Fig. 1) where $N$ is the number of nodes and $M$ is the number of wavelengths $(N \geq M)$. The $M$ distinct wavelengths create

Manuscript received July 21, 1992; revised November 9, 1992.

J. H. Laarhuis is with the Department of Electrical Engineering, University of Twente, 7500 AE Enschede, The Netherlands.

A. M. J. Koonen is with AT\&T Network Systems Netherlands and parttime with the Department of Electrical Engineering, University of Twente, The Netherlands.

IEEE Log Number 9207739.

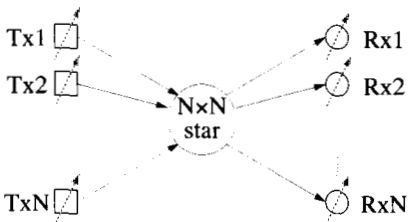

Fig. 1. Generic architecture of the multichannel star network. The number of wavelengths $M \leq N$. The dotted arrow indicates that the transceiver may be tunable or fixed.

the multiple communication channels and are given by the collection $\mathcal{M}=\left\{\lambda_{1} \cdots \lambda_{M}\right\}$. Due to the limited tunability, the sets of wavelengths where node $i$ can transmit and receive on are subsets of $\mathcal{M}$ and are denoted as $\Lambda_{i}^{t} \subset \mathcal{M}$ and $\Lambda_{i}^{r} \subset \mathcal{M}$, respectively. Each node transmits and receives only one wavelength at a time. The set of wavelengths on which a transmission from node $i$ to node $j$ can take place is formed by the cross section of $\Lambda_{i}^{t}$ and $\Lambda_{j}^{r}$ and is defined as $\Lambda_{i j} \triangleq \Lambda_{i}^{t} \cap \Lambda_{j}^{r}$. Whether or not node $i$ is allowed to transmit to node $j$ at time instant $\tau$ ( $\tau$ refers to a certain time slot in slotted systems) is given by the function $\operatorname{tr}(i, j \mid \tau)$, which can take the values ' 1 ' and ' 0 .' Finally, the network multiplicity is defined as $\Gamma \triangleq N / M$, i.e., the number of transmitters and number of receivers with identical sets of wavelengths.

Following this network setup, two network categories can be discerned depending on how the multiple transmission channels are allocated to the nodes. These are those using the real time arbitration strategy, which is essentially a demand assignment (DA) strategy, and those using the pre-defined allocation strategy which can be considered as a fixed assignment (FA) strategy.

In access mechanisms based on the real time arbitration strategy, resources are allocated to the nodes on request. This, however, requires a pre-transmission coordination to inform the intended receiver about 1) the upcoming arrival of a message, 2) the node who sends the message and 3) the wavelength where the message will be transmitted on. This coordination usually takes place on a separate control channel using minipackets which contain the abovementioned necessary information. Because more than one node may claim the same resources at a time, there is a possibility of conflicts. Therefore measures have to be taken to resolve these conflicts. Examples of these kind of access mechanisms can be found in $[2]-[5]$.

In access mechanisms based on the pre-defined allocation strategy on the other hand, resources are allocated to nodes 
whether or not they have messages to be transmitted. This is achieved by means of a scheduling algorithm. Although this implies some waste of bandwidth the advantage of the strategy is that it can be made contention-free because the mechanism is deterministic, i.e., the allocation of resources to the nodes is known in advance. Furthermore, transfer delays are bounded and there is inherent addressing, which is enabled by the scheduling algorithm. By full inherent addressing it is meant that a particular combination of wavelength and time instance provides sufficient information for a receiver to decide whether a transmission is destined for it or not. With partial inherent addressing the wavelength-time combination is not unique for a certain receiver. Therefore additional addressing has to be inserted in the message stream. In [6], [7], (partly) [8] and [9], access protocols are described which are based on the pre-defined allocation strategy.

Recently, a survey on access protocols for multichannel networks has appeared [10].

This paper is devoted to the class of access mechanisms based on the pre-defined allocation strategy which has some advantageous aspects for star based networks as will be outlined later. A number of desirable properties of multichannel networks can be identified concerning interconnectivity, access control and concurrency.

Within the network, full interconnectivity must be provided, which is a prerequisite for any network. Each node $i$ must be able to transmit to every node $j$ which is mathematically equivalent to stating that the cross section $\Lambda_{i j}$ is never empty:

$$
\Lambda_{i j} \neq \emptyset, \forall i, j \in\{1, \cdots, N\} .
$$

In multichannel networks, two kinds of contention conflicts may occur: inter-channel conflicts and intra-channel conflicts. They occur when two or more nodes send simultaneously to the same destination on different wavelengths and on the same wavelength, respectively. In the former, because the receiver can tune to only one channel at a time, one or more packets are mis-routed and are thus discarded. In the latter (intrawavelength conflicts) all packets overlap (partly) in time and are thus all destroyed. Both situations require retransmissions.

The inefficiency of feedback is dictated by the value of the normalized propagation delay $a$. This normalized propagation delay is defined as the quotient of the propagation delay and the packet transmission timie, $\tau_{d} / T_{p}$, and it is a measure of the number of packets pipelined in the network. In optical networks the value of $a$ can be much larger than one due to the high data rates possible (low dispersion of fibre) and the large network spans possible (low attenuation of fibre). Because contention-based access protocols necessitate feedback, like monitoring of transmissions and reception of acknowledgments, they lead to both inefficient use of the bandwidth and high transfer delays. So it is clear that in order to be efficient, the access mechanism should be contention-free and feedbackless whenever $\boldsymbol{a}$ is large. Therefore, real time arbitration mechanisms should be avoided if $\boldsymbol{a}$ is large. Contention-free access is attained when no simultaneous transmissions can occur to any node, i.e.:

$$
\begin{aligned}
& \operatorname{tr}(i, j \mid \tau) \cdot \operatorname{tr}(k, j \mid \tau)=0, \\
& \forall k \neq i ; \quad i, j, k \in\{1, \cdots, N\}
\end{aligned}
$$

where it is assumed that the propagation delay from a node to the star and visa versa is the same for each node.

Finally, in order to increase the throughput, the concurrency, i.e., the number of disjunct communication paths at each time instance, must be maximized. This is achieved when all $M$ wavelengths are used for simultaneous transmission between $M$ disjunct transmitter-receiver pairs. The selection of $M$ disjunct transmitter-receiver pairs $\left(i_{p}, j_{p}\right)$ is given by the expression:

$$
\prod_{p=1}^{M} \operatorname{tr}\left(i_{p}, j_{p} \mid \tau\right)=1, i_{p}, j_{p} \in\{1, \cdots, N\}
$$

where $i_{p}$ and $j_{p}, p=[1, \cdots, M]$ each denote $M$ elements drawn from the set of $N$ elements without replacements. The use of different wavelengths for the $M$ transmissions is mathematically stated by:

$$
\Lambda_{i j} \cap \Lambda_{k l}=\emptyset, \forall k \neq i, l \neq j .
$$

To increase the concurrency, $M$ must become larger. Unfortunately, with today's transceivers, this can only be accomplished at the expense of tuning speed. Examples are the acousto-optic tunable and mechanically tunable external cavity lasers [11], [12]. These lasers can be tuned over the entire gain spectrum of the laser diode. However, their tuning speed is limited to a few $\mu$ s for the acousto-optic tunable laser and, because of piezo-electric tuning, to a few $\mathrm{ms}$ for the mechanically tunable laser. Although the recently reported $\mathrm{YCC}^{3}{ }^{3}$ laser [13] might offer both wide tuning range and fast switching between wavelengths, its use in multichannel networks is hampered by the inherent complexity of its adjustment.

In the next section, a system concept will be shown that increases $M$ while using transceivers which do not suffer from the abovementioned problems and which do not require tuning over the whole set of $M$ wavelengths.

\section{THE 'STRIDIS' SYSTEM CONCEPT}

The architecture of the physical layer is an integral part of the suggested access strategy. This strategy, which is based on reduction of complexity, consists of two stages or levels. In stage one the network is divided into several disjunct subnetworks on a wavelength basis which are subsequently reconfigured on a time basis. The second stage involves employment of a simple access mechanism within each subnetwork. The second stage is treated in Section IV. In this section, first the architecture of the physical layer is described. Then the interconnection strategy of the subnetworks will be described. Finally, a method is given to control the wavelengths in the network and to achieve synchronization. 


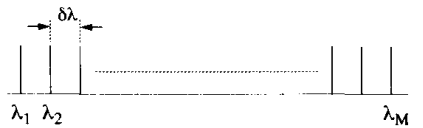

(a)

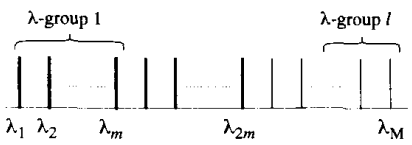

(b)

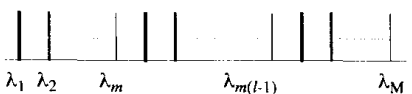

(c)

Fig. 2. Allocation of the available $M$ wavelengths (a). In (b) the wavelengths accessible by different laser transmitter modules are given by the different shades. The same is done for the wavelengths accessible by receiver modules in (c).

\section{A. Physical Layer Architecture}

In the proposed system each node is equipped with a transmitter module and a receiver module. The available $M$ wavelengths are equidistantly spaced by $\delta \lambda$ (Fig. 2(a)).

A transmitter module consists of a tunable laser which can transmit on $m$ different, consecutive wavelengths, constituting a wavelength group (Fig. 2(b)). If there is a total of $M$ wavelengths, then $M / m \triangleq l$ different transmitter modules exist and $N / l$ nodes use the same type of transmitter module.

The set of wavelengths where node $i$ can transmit on is given by

$$
\Lambda_{i}^{t}=\lambda_{((i-1) \operatorname{div}(N / l)) m+\nu} ; i=1, \cdots, N, \nu=1, \cdots, m .
$$

In (5) the expression $((i-1) \operatorname{div}(N / l))=\rho-1$ yields those nodes belonging to wavelength group $\rho$, written in short as $\lambda g_{\rho}$. The counter $\nu$ denotes the wavelength number within each $\lambda g_{\rho}$.

As an example, (5) has been applied to a $N=32$ node network using a total of $M=16$ wavelengths. Therefore, the network multiplicity $\Gamma=N / M=2$. The transmitter modules can tune to $m=4$ wavelengths. From this it follows that $l=M / m=4$ different transmitter modules are required. Table I(a) shows the allocation of the different wavelength sets to the nodes.

A receiver module consists of a wavelength demultiplexer with $l$ outlets. By means of switching, one outlet at a time is selected for reception on the corresponding wavelength (Fig. 3). To achieve full interconnectivity, each outlet carries an element of a different wavelength group.

The wavelengths of the different receiver modules are interleaved, as can be seen in Fig. 2(c). There can be precisely $m$ disjunct receiver modules which fulfil the interconnection requirement in the abovementioned way. Therefore, $N / m(=\Gamma l)$ nodes have the same receiver configuration. In a similar way

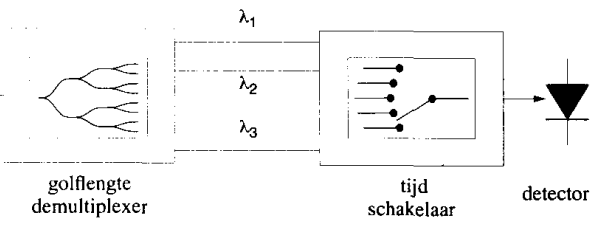

Fig. 3. Architecture of the receiver module for each node. In the figure is $\nu=1 . \cdots, m$ and $l$ is the number of outlets of the wavelength demultiplexer.

TABLE $\mathbf{I}$

Sets of Wavelengths Where Transmitters, Table (a), and Receivers, Table (b), can Tune to for a 32 Node Network With a Total of 16 Wavelengths and Each Transmitter MOdule AND Receiver MOdule CAN TUNE to 4 WaVelengths

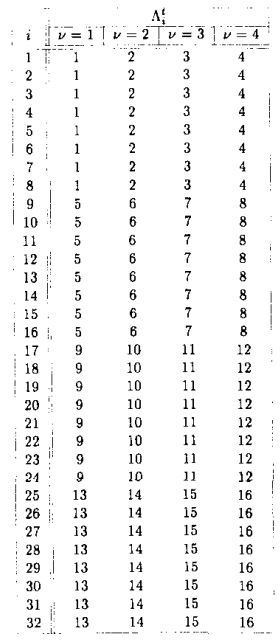

(a)

\begin{tabular}{|c|c|c|c|c|}
\hline t & $\theta=1$ & $p=2$ & $i$ & $\rho=4$ \\
\hline $1 \pi$ & 1 & 5 & 9 & 13 \\
\hline 2 & 1 & & 9 & 13 \\
\hline 3 & 1 & ป & 9 & 13 \\
\hline & & 5 & 9 & 13 \\
\hline 6 & $\frac{1}{1}$ & $\begin{array}{l}5 \\
5\end{array}$ & $\begin{array}{l}9 \\
9\end{array}$ & $\begin{array}{l}13 \\
13\end{array}$ \\
\hline & 1 & 5 & 9 & 13 \\
\hline 8 & 1 & 5 & 9 & 13 \\
\hline 9 & 2 & 6 & 10 & 14 \\
\hline 10 & 2 & 6 & 10 & 14 \\
\hline 12 & 2 & 6 & 10 & $\begin{array}{l}14 \\
14\end{array}$ \\
\hline 13 & 2 & 6 & 10 & 14 \\
\hline 14 & 2 & 6 & 10 & 14 \\
\hline 13 & ${ }^{2}$ & 6 & 10 & 14 \\
\hline 10 & 2 & 8 & iv & \\
\hline & 3 & $\therefore$ & 11 & 15 \\
\hline $\begin{array}{l}10 \\
19\end{array}$ & 3 & $=$ & 11 & 15 \\
\hline 20 & 3 & 7 & 11 & 15 \\
\hline 21 & 3 & 7 & 11 & 15 \\
\hline & 3 & 7 & 11 & 15 \\
\hline 23 & 3 & 7 & 11 & 15 \\
\hline & 3 & 7 & 11 & 15 \\
\hline 25 & 4 & 8 & 12 & 16 \\
\hline & 4 & 8 & 12 & 16 \\
\hline 21 & 4 & $\therefore$ & 12 & 16 \\
\hline 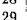 & $\begin{array}{l}4 \\
4\end{array}$ & 8 & $\begin{array}{l}12 \\
12\end{array}$ & 16 \\
\hline 30 & 4 & 8 & 12 & 16 \\
\hline & 4 & 0 & 12 & 16 \\
\hline 32 & 4 & 8 & 12 & \\
\hline
\end{tabular}

(b) as with the transmitter, $\Lambda_{i}^{r}$ is given by:

$\Lambda_{i}^{r}=\lambda_{(\rho-1) m+(i-1) \operatorname{div}(N / m)+1} ; i=1, \cdots, N, \rho=1, \cdots, l$.

It follows from (6) that the filters (wavelengths) of a receiver module are equidistantly spaced by $m \delta \lambda$ (Fig. 2(c)). As an example, for the same parameter values as for the transmitter, the allocation of sets of receiving wavelengths to the nodes has been calculated with (6) and is shown in Table I(b).

\section{B. The Interconnection Strategy}

Besides the transmitter and receiver configuration as given above, one additional thing is needed in order to satisfy the multichannel requirements given by (1)-(3) namely the existence of $l$ contiguous time slots, defined as a cycle. Within each time slot the network is interconnected differently. The receivers are responsible for the reconfiguration of the network. This is accomplished by cyclicly switching to the next outlet of the wavelength demultiplexer at the beginning of a time slot.

An important consequence of the time slotting is the fact that $\Lambda_{i}^{r}$ is now a function of the time slot number and comprises only one element at each time slot. The time dependent 
TABLE II

Time D ependent Allocation of Receiver Wavelengths to the Nodes . The Horizontal Entries are the Different Nodes, the Vertical Entries the Time Slots. The Elements of the Table are the Corresponding W avelengths. Note that the Duration of a CyCle is 4 Time Slots

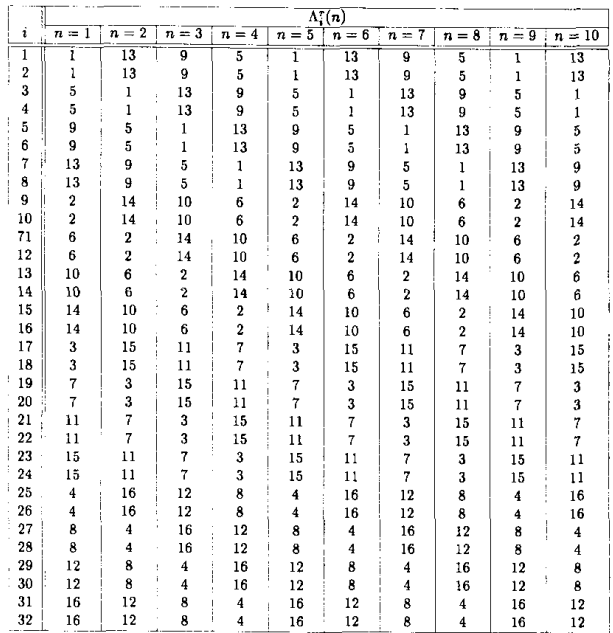

receiver wavelength allocation follows partly from (6) by substituting the time slot number $n$ for $\rho$ :

$$
\begin{aligned}
\Lambda_{i}^{r}(n) & =\lambda_{\{(n(l-1)+(i-1) \operatorname{div} \Gamma+1) \bmod l\} m+(i-1) \operatorname{div}(N / m)+1} \\
i & =1, \cdots, N, n=1, \cdots, \infty .
\end{aligned}
$$

Compared to (6), there are two differences apart from the substitution of $n$ for $\rho$. Firstly, the selection of $\Gamma$ nodes having the same receiver wavelength in a time slot, given by the expression $(i-1)$ div $\Gamma$. Secondly, the modulo operator $\bmod$, which reflects the fact that the time dependent allocation of receiver wavelengths to the nodes is periodic in $n$, with a period length $l$. In Table II this allocation is shown for the first ten time slots where (7) is used for the evaluation.

The whole of $\Lambda_{i j} \mathrm{~s}$ in the different time slots is called the interconnection scheme. For the network under consideration, this scheme is created by taking the cross section of $\Lambda_{i}^{t}$ and $\Lambda_{j}^{r}(n)$. This cross section yields those nodes sharing the same wavelength in time slot $n$. The result of this operation is that the set of nodes $T$ transmitting on wavelength group $\rho$ are scheduled for transmission in time slot $n$ to a specific set $R(\rho, n)$ of nodes, i.e.,:

$$
\begin{aligned}
& T(\lambda g) \stackrel{n}{\longrightarrow} R(\rho, n) \\
& R(\rho, n)= \Gamma[(\rho-1)+(n-1) \bmod l+l(k \operatorname{div} \Gamma)] \\
&+k \bmod \Gamma+1 \\
& k= 0,1, \cdots, \Gamma_{m}-1
\end{aligned}
$$

where $T$ and $R$ denote the set of transmitting nodes and the set of receiving nodes, respectively. The wavelengths where the nodes $i \in T$ transmit on are unambigously determined by the wavelength group $\lambda g_{\rho}$. Therefore, $R(\rho, n)$ can be considered as the interconnection matrix. This is a $[l \times l \times N / l]$-matrix, with $l$ being the number of distinct wavelength groups and time slots and $N / l$ the number of nodes in each particular set $R(\rho, n)$. The wavelengths associated with these nodes $i \in R(\rho, n)$ can be found by substituting $n$ and the values of $R(\rho, n)$ in (7).

By the choice of the transmitter and receiver configuration, the interconnection scheme creates $l$ disjunct subnetworks in a time slot. At the beginning of each next time slot these subnetworks are reconfigured to create $l$ different subnetworks. This is the kernel of the proposed access strategy, which is called 'Slotted Time Reconfigurable Interconnection of DIsjunct Subnetworks' ('STRIDIS').

Each subnetwork consists of $N / l=\Gamma m$ transmitting nodes and another $N / l$, generally not the same, receiving nodes. Thus, within one time slot, a node may have a transmit function in one subnetwork and a receiver function in another (see Table I and II). Each $l$ time slots, defined as the cycle time $T_{\text {cycle }}$, the same subnetworks is created.

The strategy assures full interconnectivity within one cycle and maximizes the concurrency by suitably configuring the transmitter/receiver multiplicity, i.e., the number of nodes having the same transmitter/receiver module. This is accomplished by maximizing the number of subnetworks according to:

$$
\begin{aligned}
& \Lambda_{i}^{t} \cap \Lambda_{j}^{t}=\emptyset,\left\{\forall i, j \mid a b s(i-j)>\frac{N}{l}\right\} \\
& \Lambda_{i}^{r} \cap \Lambda_{j}^{r}=\emptyset,\left\{\forall i, j \mid a b s(i-j)>\frac{N}{m}\right\}
\end{aligned}
$$

which is fulfilled by (5) and (6), and reducing the receiver multiplicity $N / m=\Gamma l$ by dividing it over the $l$ time slots, i.e.:

$$
\Lambda_{i}^{r}=\Lambda_{j}^{r},\left\{\forall i, j \mid 0<(j-i)<\Gamma \quad \text { and } \quad \frac{j}{\Gamma} \in I N^{+}\right\}
$$

which is implicitly fulfilled by (7).

For the same parameters as in the aforementioned example, the interconnection matrices for $\lambda g_{1}$ and $\lambda g_{2}$, i.e., $R(1, n)$ and $R(2, n)$, are shown in Table III. The matrices have been generated by (8). Each column denotes the set of nodes connected with the nodes of $\lambda g_{\rho}$ in time slot $n$. The corresponding receiver wavelengths can be found in Table II.

In addition, an illustration of the interconnection scheme is given in Figs. 4-5. In Fig. 4 the interconnection scheme for the four subnetworks is given for time slot 1 . The different shades of the arrows indicate the different subnetworks. Fig. 5 shows the interconnection scheme of $\lambda g_{1}$ for the first two timeslots. The interconnection for the remaining time slots can be deduced from the cyclic behavior of the scheme or by inspection of Table III.

The transmitter modules in the 'STRIDIS' concept can be implemented by fast, continuously tunable DFB or DBR lasers, which cover an entire wavelength group. The continuous tunability is a desirable property when used in multichannel networks because of the absence of mode-hopping and the simplicity of current adjustment (tuning). Continuous tuning ranges of 3.3 and $4.4 \mathrm{~nm}$ have been reported for a double section DFB and a DBR laser, respectively [12]. However, a continuous tuning range of $7 \mathrm{~nm}$ is expected for the tunable twin-guide (TTG) DFB laser [14]. DFB devices have the potential of faster wavelength switching than DBR devices 


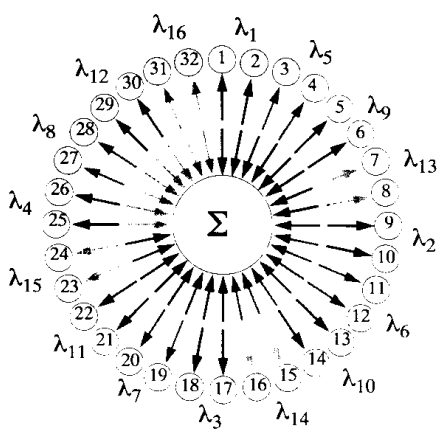

Fig. 4. Interconnection scheme for all subnetworks, distinguished by different arrow shades, for time slot 1 . Inward pointing arrows denote a transmit function and outward pointing arrows a receiving function. Note that a node generally has its transmit function in a different subnetwork than its receive function.
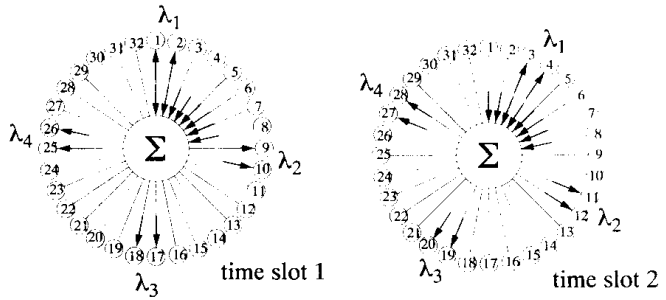

Fig. 5. Interconnection scheme for subnetwork 1 for two time slots.

TABLE III

Interconnection Matrices $R(1, n)$ AND $R(2, n)$. The Corresponding WAVELENGTHS CAN BE FOUND BY INSPECTION OF TABLE II

\begin{tabular}{|c|c|c|c|}
\hline \multicolumn{4}{|c|}{$R(\mathbf{1}, \mathbf{n})$} \\
\hline$n=1$ & $\mathbf{n = 2}$ & $\mathbf{n = 3}$ & $\mathbf{n = 4}$ \\
\hline \hline 1 & $\mathbf{3}$ & 5 & 7 \\
2 & 4 & 6 & $\mathbf{8}$ \\
9 & $\mathbf{1 1}$ & $\mathbf{1 3}$ & 15 \\
10 & $\mathbf{1 2}$ & $\mathbf{1 4}$ & 16 \\
17 & $\mathbf{1 9}$ & 21 & 23 \\
18 & 20 & 22 & $2 \mathbf{4}$ \\
25 & 27 & 29 & 31 \\
26 & $\mathbf{2 8}$ & $\mathbf{3 0}$ & $\mathbf{3 2}$ \\
\hline
\end{tabular}

\begin{tabular}{|c|c|cc}
\hline & \multicolumn{3}{c}{$R(2, n)$} \\
\cline { 3 - 4 } & $n=2$ & $n=3$ & $n=4$ \\
\hline \hline 3 & 5 & 7 & 1 \\
4 & 6 & 8 & 2 \\
11 & 13 & 15 & 9 \\
12 & 14 & 16 & 10 \\
19 & 21 & 23 & 17 \\
20 & 22 & 24 & 18 \\
27 & 29 & 31 & 25 \\
28 & 30 & 32 & 26 \\
\hline
\end{tabular}

because of the shorter carrier lifetimes in active material due to stimulated emission [15]. Up till now, for both types of devices tuning times have been reported, which are lower than 5 ns [15], [16]. Although the tuning range of the individual devices is not large, the total wavelength range, and accordingly the total number of wavelengths in the network can be much larger using the 'STRIDIS' concept.

The receiver modules can be implemented by MachZehnder interferometric 1-to- $l$ filters [17]. Switching between the filters can be achieved by fast optical space switches. The advantage of this implementation is the absence of a $1 / l$ splitting loss and the possibility to integrate it on electrooptic materials. Other implementations of this filter configuration are being studied.

Although the 'STRIDIS' concept has been illustrated for a specific example, it is a generic concept. Different networks can be created using the 'STRIDIS' concept, depending on the specific values of the integer valued parameters $l$, being the number of subnetworks or wavelength groups, and $m$, the number of wavelengths in a wavelength group. These parameters can create the combinations $\{(l, m) \mid l \leq M, l m=$ $M\}$, which in the following will be referred to as the $(l, m)$ configurations.

Consider for instance the number of nodes to be equal to the number of wavelengths, $N=M$, and the number of subnetworks $l=N$. Then there are $l=N$ different transmitter modules, each having a range of $m=1$ wavelengths. So each node has its unique transmitter wavelength. There are $m=1$ different receiver modules, each able to tune to $l=N$ wavelengths. So all nodes can receive on all different wavelengths. The configuration thus created, $(N, 1)$, is the well-known 'Broadcast \& Select' architecture [18]. The other extreme, namely the 'Wavelength Routing' architecture [18] with transmitters tunable to all different wavelengths and each node having a unique receive wavelength, can also be created with 'STRIDIS' by choosing $m=N=M$ thereby creating the $(1, N)$ configuration.

\section{Wavelength and Time Management}

To account for wavelength misalignments some kind of wavelength control must be employed. In the following a wavelength control procedure will be described, in which first the wavelengths of the receiver filters are controlled and next the laser wavelengths are adjusted to the controlled filter wavelengths.

A close look at Fig. 2(b) and (c) reveals that any tunable transmitter laser can reach precisely one wavelength position of each different multiwavelength-pass receiver filter. If it is possible to control all wavelengths of a receiver filter simultanously by locking only one of its wavelengths to a reference, the laser of a single station can serve as a master for the filter wavelengths of the whole network. Filters based on the interferometric principle offer this possibility: they have a transmission characteristic which is periodic in optical frequency. The two parameters which determine the situation of the resonance wavelengths $\lambda_{k}$ are given by:

$$
\begin{gathered}
\lambda_{k}=\frac{2 n L}{k} \\
\Delta \lambda_{k} \triangleq \lambda_{k}-\lambda_{k+1}=\frac{\lambda_{k}}{k+1} .
\end{gathered}
$$

where $n L$ is the optical path length in the filter ( $n$ the refractive index and $L$ the geometric distance) and $k$ is an integer. If the variation in a resonance wavelength is zero, then the adjacent wavelengths exhibit no variations, too. This means that the whole comb of resonance wavelengths of a filter can be controlled by locking only one resonance wavelength.

The locking on a specific wavelength can be achieved by tapping a small amount of power containing the specific wavelength, and maximizing this power by tuning the filter via a feedback circuit. The specific wavelength signal is selectively filtered out by the feedback circuit which detects the unique identification signal superimposed on the data signal at the master laser (Fig. 6). In this way false locking of the receiver filter is avoided. In service and continuous control are important features of this control method.

Adjustment of the laser wavelength positions to the filter wavelengths is accomplished by back-looping at each node. In 


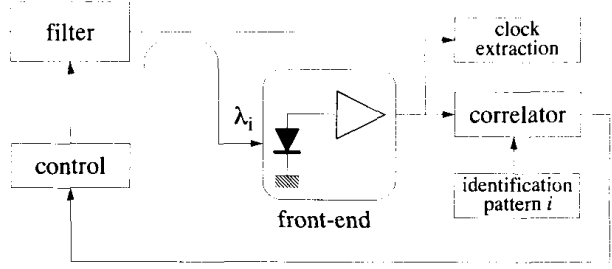

Fig. 6. Schematic overview of the control circuit for wavelength control and network synchronization.

each cycle there exists a subslot $n_{s}$ for which $\operatorname{tr}\left(i, i \mid n_{s}\right)=1$. In this subslot station $i$ can adjust one wavelength of its laser to its receiver filter. By maximizing the received power, the laser wavelength position is optimally adjusted. Moreover, assuming that the remaining wavelengths of that laser can be adjusted correctly given one wavelength is correct, all laser wavelengths are controlled.

Because there are $m$ different receiver filters, there also have to be $m$ master laser wavelengths imposing the reference wavelengths (e.g., a master wavelength comb containing $m$ reference wavelengths).

For proper operation of the network, all receiver filters and transmitter lasers must retune at the same time instances. Therefore the network needs to be synchronized. Synchronization can be achieved by distribution of a common clock signal. This clock signal can continuously be superimposed on each data signal of the abovementioned master node. Each node can then extract the clock signal using the control circuit of Fig. 6 .

\section{Performance Analysis}

According to Section II, a contention-free access mechanism is employed within each subnetwork. The mechanism is based on a combination of time-division and wavelength division (quasi-wavelength routing). This implies that each time slot has to be divided into several subslots. The duration of a subslot is denoted by $T_{s u b}$. As a consequence of this approach, the physical layer hardware largely takes care of the contention resolution. The advantage of this approach is the reduction of protocol processing because the inherent addressing, routing and flow control of the physical layer need not be performed by higher layer protocols, whose processing times are obviously slower than the hardware reconfiguration times.

For our analysis the following performance metrics are important. The capacity of the network is the maximum number of bits per second which can enter the physical layer. The throughput is defined as the average number of databits transferred by the network per second. Because there is no contention, the throughput equals the offered load as long as this load does not exceed the capacity. For high loads the throughput converges asymptotically to the capacity. A node is defined as a system comprising a waiting queue and a server. The utilization is the fraction of the time a server is busy. The average transfer delay of a message is the average time a message experiences from entering the queue of an originator till the complete reception of the message at a destination. The average transfer delay depends largely on the time spent in the queue and the service time of the message. The propagation delay can be added as a constant to the average transfer delay. All aspects subject to source node $i$ and destination node $j$ are indicated by $(i, j)$ (e.g., message $(i, j)$, queue $(i, j)$ et cetera). The traffic offered to node $i$ for transmission is called perfectly symmetric if the traffic intensity $(i, j)$ equals the traffic intensity $(i, k),(\forall j \neq k)$.

In the first part of this section, the protocol employed within the subnetworks will be described and analyzed. Hereafter the performance of the network is evaluated.

\section{A. Generic Wavelength-Time Protocol}

A generic wavelength-time allocation $(w t a)$ protocol on subnetwork level can be created by defining three parameters which can be varied to adjust the protocol:

1. each time slot is divided into $q$ subslots. In a subslot, precisely one packet can be sent per wavelength.

2. within each subslot, $p$ concurrent transmissions can take place, Because there are $m$ different wavelengths within each subnetwork we have $1 \leq p \leq m$.

3. per subslot, each transmitter has $h$ possible destinations to choose from. Actually, this means that the queues for $h$ destinations are combined. Clearly $1 \leq h \leq \Gamma m$ because each transmitter can tune to all wavelengths in a subnetwork and is therefore able to reach any receiving node in that subnetwork.

To assure full interconnectivity within the subnetwork, it must be possible to create $(N / l)^{2}=(\Gamma m)^{2}$ different transmitter-receiver pairs within the lifetime of the subnetwork, which is one time slot. Therefore, the product of the three parameters must equal the number of different combinations, i.e.:

$$
p q h=(\Gamma m)^{2}
$$

When the protocol fulfils (13), the full interconnectivity demand for each subnetwork is satisfied and therefore also the requirement for full interconnectivity of the whole network, (1). Besides this requirement, some performance demands can be implied on the $w t a$-protocol. The protocol will be chosen which has the lowest delays and the highest throughput for most kinds of traffic. Three different values for the parameter $h$ can be identified which reflect specific situations:

$w t a$-protocol 1, $h=1$ : In this protocol, each disjunct transmission opportunity (whose total is determined by the number of subslots $q$ times the concurrency $p$ ) within a subnetwork is allocated to a unique transmitter-receiver pair. Because all $m$ wavelengths of a subnetwork can be used for concurrent transmissions, it follows with (13) that the number of subslots equals $\Gamma^{2} m$. With $h=1$ no addressing need to be provided by the transmitter because this is fully determined by the wavelength-time allocation. When all nodes have high loads, this protocol is suitable to process symmetric traffic. As an example, $w t a$-protocol 1 has been applied to the network earlier used as an example in Section III. For time slot 1 of subnetwork 1, which is situated in Fig. 5, Table IV shows the wavelength-time allocation. 
TABLE IV

allocation of the Transmission Resources, l, E., Wavelength and Time to THE SPECIFIC Transmitters and ReCEIVERS For $w$ t $a$-Protocol $1(h=1)$ on Subnetwork Level. The Variable $n_{s}$ Denotes the Subtimeslots, in Contrast to the Timeslots $n$. This Subnetwork Allocation has been Worked OUt for Subnetwork 1 In Time Slot 1, as Shown by Fig. 5

\begin{tabular}{|c|c|c|c|c|c|c|c|c|}
\hline & \multicolumn{8}{|c|}{$\operatorname{tr}\left(i, j \mid n_{i}\right)$ for $\lambda g_{1}$ with wta-protocol 1} \\
\hline$\frac{\lambda}{\lambda}$ & $n=1$ & $n_{c}=2$ & $n_{0}=3$ & $n_{0}=4$ & $n_{s}=5$ & $n_{0}=6$ & $=n_{s}=7$ & $\frac{n}{4}=8$ \\
\hline$\lambda_{2}$ & $\begin{array}{l}1 \rightarrow 1 \\
2 \rightarrow 8\end{array}$ & $\lim _{2 \rightarrow 9}$ & $2 \rightarrow 1$ & $\begin{array}{l}2 \rightarrow 2 \\
3 \rightarrow 9\end{array}$ & $3 \rightarrow 1$ & $\begin{array}{l}3 \rightarrow 2 \\
4 \rightarrow 9\end{array}$ & $4 \rightarrow 1$ & $\begin{array}{l}4 \rightarrow 2 \\
5 \rightarrow 9\end{array}$ \\
\hline & $3 \rightarrow 17$ & $3 \rightarrow 18$ & $4 \rightarrow 17$ & $4 \rightarrow 18$ & $5 \rightarrow 17$ & & $6 \rightarrow 17$ & \\
\hline$\lambda_{1}$ & $4 \rightarrow 25$ & $4-26$ & $5 \rightarrow 25$ & $5 \rightarrow 26$ & $6 \rightarrow 25$ & $8 \rightarrow 26$ & $7 \rightarrow 25$ & $7 \rightarrow 26$ \\
\hline$\lambda$ & $n_{z}=9$ & $n_{1}=10$ & $n_{1}=11$ & $n_{4}=12$ & $n,=13$ & $n_{1}=14$ & $n_{0}=15$ & $n_{x}=16$ \\
\hline$\lambda_{1}$ & $5 \rightarrow 1$ & $5-2$ & $6 \rightarrow 1$ & 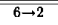 & $7 \rightarrow 1$ & 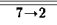 & $8 \rightarrow 1$ & $\overline{8 \rightarrow 2}$ \\
\hline$\therefore$ & $6 \rightarrow 8$ & $6 \rightarrow 9$ & 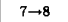 & $7 \rightarrow 9$ & $8 \rightarrow 8$ & & $1 \rightarrow 8$ & $1 \rightarrow 9$ \\
\hline$\lambda_{3}$ & $7 \rightarrow 17$ & $7 \rightarrow 18$ & 17 & 18 & $1 \rightarrow 17$ & 18 & $2 \rightarrow$ & \\
\hline$\lambda_{1}$ & $8 \rightarrow 25$ & & $1 \rightarrow 25$ & $1 \rightarrow 26$ & $\rightarrow 25$ & $2 \rightarrow 26$ & $3 \rightarrow 25$ & $3 \rightarrow 26$ \\
\hline
\end{tabular}

TABLE $\mathrm{V}$

SAme as in TABle IV but NOW fOR $u+a$-Protocol $2(h=\Gamma)$

\begin{tabular}{|c|c|c|c|c|c|c|c|c|}
\hline & \multicolumn{8}{|c|}{$\operatorname{tr}(i$} \\
\hline$\lambda$ & $n_{e}=1$ & $n_{\bullet}=2$ & $n_{t}=3$ & $n_{i}=4$ & $n_{s}=5$ & $n,=6$ & & \\
\hline & $1 \rightarrow 1,2$ & $=1, \overline{2}$ & $3 \rightarrow 1,2$ & $4 \rightarrow 1$ & $5 \rightarrow 1,2$ & & & \\
\hline & $2 \rightarrow 8,9$ & $-8,9$ & $4 \rightarrow 8,9$ & $5 \rightarrow 8$, & $6 \rightarrow 8,9$ & $7 \rightarrow 8,9$ & $8 \rightarrow 8,9$ & 9 \\
\hline$\lambda_{3}$ & $\begin{array}{l}3 \rightarrow 17,18 \\
4 \rightarrow 25,26\end{array}$ & $4 \rightarrow 17,18$ & $5 \rightarrow 17,18$ & $6 \rightarrow 17,18$ & $7 \rightarrow 17,18$ & $8 \rightarrow 17,18$ & $1 \rightarrow 17,18$ & 18 \\
\hline & 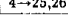 & $5 \rightarrow 25,26$ & $6 \rightarrow 25,26$. & $7 \rightarrow 25,26$ & $8 \rightarrow 25,26$ & $\rightarrow 25,26$ & $2-25,26$ & -25 \\
\hline
\end{tabular}

TABLE VI

Same as in Table IV, but now for $u t a$-Protocol $3(h=\Gamma m)$. This TABle EXemplifies that the Transmitting Nodes in the SubNetwork Can in Turn Select Any of the Avallable Resources in the Subnetwork $\left(\lambda g_{1}\right)$ to Send to Any of the Receiving Nodes in the Subnetwork $(R(1.1))$.

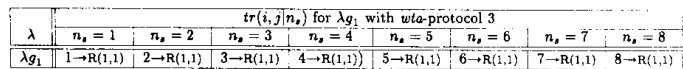

wta-protocol 2, $h=\Gamma$ : In this situation, the concurrency can still be maintained at the maximum value of $m$. However, transmitters have to add a minimum addressing to the packet headers to distinguish the $\Gamma$ nodes having the same receiver module in the same time slot (now there is no full inherent addressing anymore). This situation reflects the maximum use of resources at a minimum number of subslots, namely $q=\Gamma m$. The wavelength-time allocation of wta-protocol 2 is illustrated in Table V.

wta-protocol 3, $\boldsymbol{h}=\boldsymbol{\Gamma m}$ : Now each subslot is allocated to a specific transmitter, which can choose from all receivers where to send to. Full addressing has to be provided by the transmitters. Clearly this reflects the situation of minimum concurrency $(p=1)$ and minimum number of subslots $(q=\Gamma m)$. This protocol might be suitable for asymmetric traffic. For $w$ ta-protocol 1 this would yield low utilizations for various $n_{s}$. For $w t a$-protocol 3, however, the utilization tends to be larger and more constant, which is caused by the fact that $h$ queues are combined for each $n_{s}$. The wavelength-time allocation of $w t a$-protocol 3 is illustrated in Table VI.

It appears that $w t a$-protocol 2 is the most efficient and flexible one: most efficient because it achieves maximum resource usage at a minimum number of subslots, and most flexible because it incorporates the specific features of the other two protocols. This can be seen as follows: if each node has a packet for all destinations within one cycle, then in protocol 1 these packets are delivered in one cycle of $l \Gamma^{2} m=N^{2} / M$ subslots. In protocol 2, a transmitter can choose among $\Gamma$ destinations for each transmission, so all these packets can be delivered within $\Gamma$ cycles, each of $l \Gamma m=N$ subslots. This also leads to a total of $N^{2} / M$ subslots.
On the other hand it is obvious that wta-protocol 2 serves asymmetric traffic as well as protocol 3 . Although the utilization is smaller for each queue in protocol 2, the concurrency of $m$ wavelengths will equal the utilization of each subslot to that of protocol 3 .

This optimum case, wta-protocol 2, resembles closely the Shared Time Division protocol proposed by Ganz and Chlamtac in [7]. Now, however, the protocol is applied to a passive star topology and constitutes a sub-protocol within the 'STRIDIS' concept.

\section{B. 'STRIDIS' Performance Evaluation}

The performance of the 'STRIDIS' system is measured by the average transfer delay of a message and by the throughput of the network. Both performance metrics will be normalized with respect to the packet transmission time $T_{p}$ to abstract from variables like the bit rate and the packet length. To simplify the performance evaluation of the system, the following assumptions have been made:

- Each node is modeled by $q l M^{B}|G| 1$ queues (bulk arrivals) and the nodes are assumed to be independent.

- The average arrival rate of messages $(i, j)$ is defined as $\Psi_{i j}$ (where we deliberately depart from the convention to use $\lambda$ for this to avoid confusion with the wavelength denotation).

- The number of packets comprising a message $(i, j)$ is a random variable with first and second moments $L_{i j}^{(1)}$ and $L_{i j}^{(2)}$, respectively.

- It is assumed that traffic offered to node $i$ is perfectly symmetric, i.e., $L_{i j}^{(1,2)}=L_{i}^{(1,2)}$ and $\Psi_{i j}=\Psi_{i}, \forall j \in$ $[1, \cdots, N]$.

- The system is assumed to be stable, i.e., utilization is smaller than one.

With these assumptions, the utilization of node $i$ for a queue can be evaluated. The average number of packets $(i, j)$ arriving at a node can is given by $L_{i}^{(1)} \Psi_{i} T_{\text {cycle }}$. Because there is one packet served per cycle time, the utilization becomes:

$$
\begin{aligned}
\rho_{i}\left(n_{s}\right) & =L_{i}^{(1)} \Psi_{i} T_{\text {cycle }} \sum_{j=1}^{N} \operatorname{tr}\left(i, j \mid n_{s}\right) \\
& =\Psi_{i} h L_{i}^{(1)} T_{\text {cycle }}
\end{aligned}
$$

where the function $\operatorname{tr}\left(i, j \mid n_{s}\right)$, which gives the implicit dependence of the parameter $h$ on the utilization, was defined in Section II. The latter equation can be derived from the former by recognizing that $\Sigma_{j=1}^{N} \operatorname{tr}\left(i, j \mid n_{s}\right)=h$.

Equation (14) can be identified as a form of Little's result [19] where $\rho_{i}\left(n_{s}\right)$ gives the utilization for a queue, $\Psi_{i} h$ the arrival rate of messages for that queue and $L_{i}^{(1)} T_{\text {cycle }}$ their average service time. It can easily be derived that the second moment of this service time is given by $L_{i}^{(2)} T_{c y c l e}^{2}$.

The average transfer delay of messages, experienced at a queue of node $i$ will now be analyzed. This delay consists of two parts: the queueing delay and the service time. The queueing delay is determined by the Pollaczek-Khinchin $(\mathrm{P}-\mathrm{K})$ formula [19] and represents the average time from 


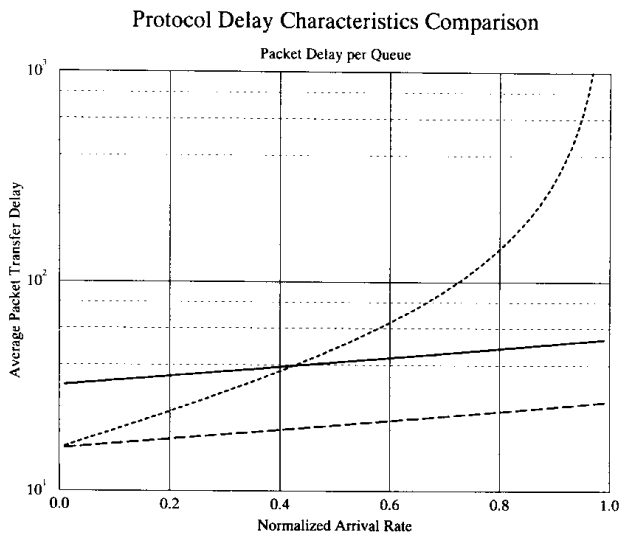

Fig. 7. Transfer delay characteristics for wta-protocol 1 (solid line), protocol 2 (dashed line) and protocol 3 (dotted line). For all protocols, the same arrival rate of packets apply. The value 1 for the normalized arrival rate corresponds to unity load for protocol 3.

the arrival of a message at the queue till it has reached the head of the queue. The service time is, on the average, half a cycle time plus a packet transmission time for the first packet of the message, and precisely one cycle time for all succeeding packets of that message. In [20] a pure TDMA system has been analyzed using the earlier mentioned assumptions. Applying these results to the 'STRIDIS' network, the following expression for $\hat{D}_{i}$, the average normalized transfer delay of a message can be derived:

$$
\hat{D}_{i}=\frac{T_{s u b}}{T_{p}}\left[\left(L_{i}^{(1)}-0.5\right) q l+1+\frac{\rho_{i}}{2\left(1-\rho_{i}\right)} \frac{L_{i}^{(2)}}{L_{i}^{(1)}} q l\right] .
$$

In (15), the product $q l$ denotes the total number of subslots within one cycle. This product, which influences strongly the delay characteristics, is determined by the specific wtaprotocol: for protocol 1 this product amounts $q l=N^{2} / M$, and for the other two $q l=N$. Although it appears that $w t a$ protocol 2 and 3 exhibit no dependence of the configuration $(l, m)$ on the transfer delay, this dependence is hidden in the load $\rho_{i}$. Assuming identical arrival processes for all three $w t a-$ protocols, the per queue load given by (14) is the same for protocol 1 and 2, but is larger for protocol 3 by a factor $m$ (from (14) it follows that $h T_{\text {cycle }}=q l h T_{s u b}=N^{2} / M T_{\text {sub }}$ for $w t a$-protocol 1 and 2 , and $h T_{\text {cycle }}=N^{2} m / M T_{s u b}$ for $w t a-$ protocol 3). For the earlier used example network, the delay characteristics for the three $w t a$-protocols have been calculated in case $L^{(1)}=L^{(2)}=1$. Fig. 7 shows these characteristics as a function of the normalized arrival rate of packets. The normalization factor equals the arrival rate which leads to unity utilization per queue for $w$ ta-protocol 3 (this renders a utilization of 0.25 for the other two protocols). The figure clearly shows that protocol 2 gives the lowest delays for the whole range of offered traffic.

For wta-protocol 1 and 2 it can be concluded that if there is any dependence of the configuration $(l, m)$ on the transfer delay, it is only the product $m l=M$ and not their individual values. Therefore, a network using a viable configuration $(l, m)$ performs as well as, e.g., the 'Broadcast

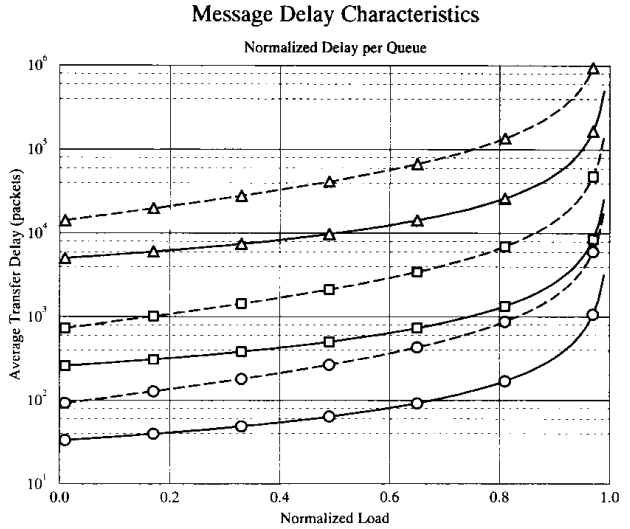

Fig. 8. Normalized average message delay as a function of the network load. The solid lines show the delay characteristics with the number of subslots per cycle being $64(0) .512(\square)$ and $10000(\triangle)$ for the case $\operatorname{Pr}[L=1]=1$. The dashed lines show the effect of bulk arrivals $\operatorname{Pr}[L=10]=0.1$, i.e., messages consisting of 10 packets arrive, on the average, each 10 messages. The same number of subslots is taken here as the parameter.

\& Select' configuration $(1, l \mathrm{~m})$ or the 'Wavelength Routing' configuration $(l m, 1)$. Although these configurations obviously are not viable for large values of $l m=M$, they are in general considered as desirable multichannel network architectures.

In Fig. 8 the average transfer delay of a message is shown as a function of the network load. These curves are valid for all $w t a$-protocols. The number of subslots $q l$ and the composition of messages in packets, expressed by $L^{(1)}$ and $L^{(2)}$, are used as parameters. For the number of subslots the values 64,512 and 10000 are assumed, which can be distinguished by the markers $\circ, \square$, and $\triangle$, respectively. The curves for the different values of $L^{(1)}$ and $L^{(2)}$ have been generated by assigning different probabilities to arrivals of messages which consist of 1 or 10 packets. The solid lines in the figure comprise the delay characteristics for $\operatorname{Pr}[L=1]=1$ leading to $L^{(1)}=1$ and $L^{(2)}=1$ and the dashed lines those for $\operatorname{Pr}[L=10]=0.1$, which yields $L^{(1)}=1.9$ and $L^{(2)}=10.9$. The values of $L$ and their probabilities have just been chosen to show the effect of bulk arrivals on the transfer delay.

The delay curves can be used as follows: based on knowledge of the arrival proces (i.e., $\Psi$, which must be symmetric for these curves, $L^{(1)}$ and $\left.L^{(2)}\right)$, select a $w t a$-protocol according to (14) such that the load per queue is smaller than 1 . Then the required number of subslots can be calculated when the total number of wavelengths $M$ (and $m$ for protocol 3) is known. The appropriate curve can now be selected. Finally, multiplication with the factor $T_{s u b} / T_{p}$ must be carried out.

The duration of one subslot, $T_{s u b}$, is determined by 1) the clockrate $R$ of the individual channels, 2) the number of bits per packet $Y$ (so the actual time to transmit a packet is $T_{p}=Y / R$ ) and 3) the duration of the guardband $(G)$. This guardband consists of two parts. First, it provides the time necessary for the tunable lasers to tune to the appropriate wavelength at the beginning of each subslot $\left(G_{\text {tune }}\right)$. At the beginning of each slot ( $\equiv q$ subslots) this time must also be long enough for the receivers to reconfigure the subnetworks. Secondly, a guardband has to be inserted to compensate for 


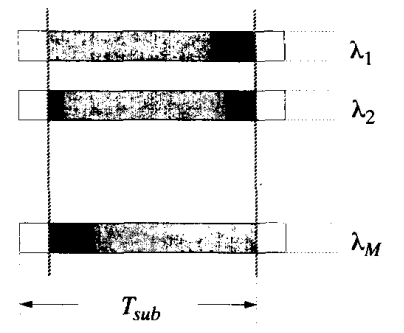

packet transmission time $T_{p}$

latency difference guardband $G_{\text {lat }}$

tuning guardband $G_{\text {tune }}$

absolute time instances for

all wavelengths

Fig. 9. Composition of a subslot. Note that $G_{l a t}$ accounts only for the latency differences, not the absolute latency.

the latency difference between packets transmitted on the outermost wavelengths $\left(G_{l a t}\right)$. This composition of a subslot is shown in Fig. 9.

For the example network, a span of $100 \mathrm{~km}$ and a maximum difference of the wavelengths $\Delta \lambda=8 \mathrm{~nm}$ (i.e., $0.5 \mathrm{~nm}$ spacing between the individual wavelengths) is assumed. Using a standard fiber with dispersion $D=16 \mathrm{ps} / \mathrm{km} / \mathrm{nm}$, the latency difference is $G_{\text {lat }} \approx 13$ ns. When using the transceivers mentioned in the previous section, $G_{\text {tune }}=5 \mathrm{~ns}$ will suffice. For the analysis we will assume that $R=1 \mathrm{~Gb} / \mathrm{s}$ and $Y=424$ bits, being the length of an ATM-cell. With these values, $T_{p}=424 \mathrm{~ns}, T_{s u b}=442 \mathrm{~ns}$ and the quotient $T_{p} / T_{s u b}$ amounts 0.96 .

The maximum throughput is easily obtained by multiplying the maximum throughput per channel by the number of simultaneous transmissions, i.e., the concurrency. The former is equal to $R T_{p} / T_{s u b}$ while the latter is $p l$, which equals $m l=M$ for the chosen $w t a$-protocol 2 . Expressed in the number of packets transmitted per packet transmit time, the maximum throughput becomes $M T_{p} / T_{s u b}$. For the example network, the non-normalized throughput at medium access control sublayer is $30.7 \mathrm{~Gb} / \mathrm{s}$.

\section{CONCLUSIONS}

In this paper, we have reported on a medium access control strategy for WDM multichannel star networks, which is based on a Slotted Time Reconfigurable Interconnection of DIsjunct Subnetworks ('STRIDIS'). Unlike other multichannel network concepts proposed, in the 'STRIDIS' concept only lasers with a limited tuning range and receivers with a limited number of integrated optics wavelength filters, both based on proven technology, are used.

The transmitted modules consist of lasers which can tune to a number of consecutive wavelengths, forming a wavelength group. The different transmitter modules form a concatenation of wavelength groups. Receiver modules consist of a set of filters, each falling within the wavelength group of a different transmitter module. The wavelengths of different receiver modules are interleaved. The creation and reconfiguration of the disjunct subnetworks is achieved by requiring that each receiver switches to a specific filter (wavelength) in each time slot. This is done in an ordered way dictated by an interconnection scheme.

The specific configurations of transmitter lasers and receiver filters allow for an efficient, in-service and continuous wavelength control method, in which only one master transmitter module controls all receiver filter wavelengths. Moreover, this master transmitter module enables network synchronization by distributing a synchronization signal on all of its reference wavelengths. Furthermore back-looping allows transmitters to be adjusted to their receiver filters.

Using the 'STRIDIS' concept, multichannel networks can be realized which provide full interconnectivity, maximum concurrency and contention free access. Furthermore, the number of nodes $N$ can be large while still having a low multiplicity $\Gamma$. The efficiency of the concept is caused by moving the higher layer protocol functions addressing, routing and flow control down to physical layer hardware. As a consequence, there is less protocol overhead which enables faster processing.

To achieve contention-free access, a generic wavelengthtime allocation protocol has been defined on subnetwork level. This protocol is characterized by the number of subslots per time slot, the subnetwork concurrency per subslot and the number of alternative destinations per transmitter per subslot. It appeared that the optimum protocol, i.e., the one having the smallest delays and the highest throughputs for all kinds of traffic, is the protocol which uses the minimum number of subslots and the maximum number of wavelengths in a subnetwork.

Due to its insensitivity to the normalized propagation constant $a$ this concept is quite suitable for large high-speed networks. Like all FA-based networks, one relatively highspeed data stream destined for a single node cannot be as efficiently handled as multiple relatively low-speed data streams, which imposes the same load, destined for different nodes. Also, the network benefits from a more steady and less bursty character of the offered traffic.

From the abovementioned, it can be concluded that the 'STRIDIS' concept will be very suitable for application in high-level broadband interconnect MAN's [21], where the nodes are essentially concentrators of the traffic offered by the lower level LAN's and MAN's.

\section{ACKNOWLEDGMENT}

The authors would like to thank A. van Moorsel and R. de Ridder for their fruitful comments on the manuscript.

\section{REFERENCES}

[1] P. E. Green, "The future of fiber-optic computer networks," IEEE Comput., vol. 25, no. 9, pp. 78-87, 1991.

[2] I. M. I. Habbab, M. Kavehrad, and C. E. W. Sundberg, "Protocols for very high speed optical fibre local area networks using a passive star topology," J. Lightwave Technol., vol. LT-5, no. 12, pp. 1782-1794, 1987. 
[3] N. Mehravari, "Performance and protocol improvements for very high speed optical fibre local area networks using a passive star topology," J. Lightwave Technol., vol. 8, no. 4, pp. 520-530, 1990.

[4] H. Shi and M. Kavehrad, "Aloha/Slotted-CSMA protocol for a very high speed optical fibre local area network using passive star topology," Proc. Infocom '91 (Ball Harbour, FL), paper 12D.4, pp. 1510-1515, 1991.

[5] G. N. M. Sudhakar, N. D. Georganas, and M. Kavehrad, "Slotted aloha and reservation aloha protocols for very high speed optical fibre local area networks using a passive star topology,"J. Lightwave Technol., vol 9, no. 10 , pp. 1411-1422, 1991.

[6] I. Chlamtac and A. Ganz, "Channel allocation protocols in frequencytime controlled high speed networks," IEEE Trans. Commun., vol. 36, no. 4 , pp. $430-440,1988$.

[7] A. Ganz and I. Chlamtac, "Path allocation access control in fibre optic communication systems," Proc. Infocom ' 88 (New Orleans, LA), paper 1B.1, pp. 0035-0044, 1988.

[8] A. Ganz and Z. Koren, "WDM passive star protocols and performance analysis," Proc. Infocom '91 (Ball Harbour, FL), paper 9A.2, pp. 0991-1000, 1991.

[9] A. Ganz and Y. Gao, "A time-wavelength assignment algorithm for a WDM star network," Proc. Infocom '92 (Florence, Italy), paper 9B.4, pp. $2144-2150,1992$.

[10] B. Mukherjee, "WDM-based local lightwave networks part 1: Singlehop systems," IEEE Network, pp. 12-27, 1992.

[11] C. A. Brackett, "Dense wavelength division multiplexing networks: Principles and applications," IEEE J. Select. Areas Commun., vol. 8, no. 6, pp. 948-964, 1990.

[12] T. P. Lee and C. E. Zah, "Wavelength tunable and single frequency semiconductor lasers for photonic communications networks," IEEE Commun. Mag., vol. 27, no. 10, pp. 42-52, 1989.

[13] M. Schilling et al., "Integrated interferometric injection laser: Novel fast and broad-band tunable monolithic light source," IEEE J. Quantum Electron., vol. 27, no. 6, pp. 1616-1623, 1991.

[14] M. C. Amann and W. Thulke, "Continuously tunable laser diodes: Longitudinal versus transverse tuning scheme," IEEE J. Select. Areas Commun., vol. 8, no. 6, pp. 1169-1177, 1990.

[15] H. Kobrinski et al., "Fast wavelength switching of laser transmitters and amplifiers," IEEE J. Select. Areas Commun., vol. 8, no. 6, pp. 1190-1202, 1990.

[16] B. Glance et al., "Multi gigabit $1 \times 10$ optical packet switching base on WDM," Proc. OFC'92 (San Jose, CA), paper ThC2, 1992.

[17] N. Takato et al, "Silica based integrated optic Mach-Zehnder multi/demultiplexer family with channel spacing of $0.01-250 \mathrm{~nm}$," IEEE J. Select. Areas Commun., vol. 8, no. 6, pp. 1120-1127, 1990.

[18] M. S. Goodman, "Multiwavelength networks and new approaches to packet switching," IEEE Commun. Mag., vol. 27, no. 10, pp. 27-35, 1989
[19] L. Kleinrock, Queueing Systems-Volume 1: Theory. New York: Wiley, 1976.

[20] S. S. Lam, "Delay analysis of a time division multiple access (TDMA) channel," IEEE Trans. Commun., vol. 25, no. 12, pp. 1489-1494, 1977.

[21] I. Rubin and J. E. Baker, "Media access control for high-speed local area and metropolitan area communications networks," Proc. IEEE, vol. 78, no. 1 , pp. $168-203,1991$.

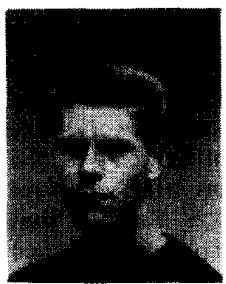

Jan H. Laarhuis was born in Boekelo, The Netherlands, on February 3, 1963. He received the M.Sc. degree in electrical engineering from the University of Twente, The Netherlands, in 1989.

In 1988 he joined the PTT Research Laboratories where his interests were the analysis, design, and realization of LiNbO\$ $3 \$$ components. In 1991 he joined the Tele-Informatics and Open Systems Group at the University of Twente to pursue the Ph.D. degree. His current interests comprise components and protocols for all optical networks.

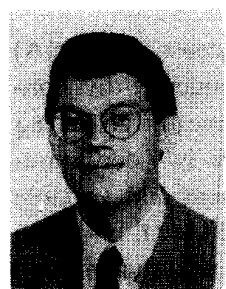

A. M. J. Koonen was born in Oss, The Netherlands, on October 20,1954 . He received the M.Sc. degree (cum laude) in electrical engineering from the Eindhoven University of Technology, The Netherlands, in 1979.

In the same year he joined the Advanced Development Transmission Department, Philips' Telecommunicatic Industrie, Huizen, The Netherlands, since 1984 part of AT\&T Network Systems Nederland. Currently, he supervises the group Optical Systems in the department Explorations. He has worked on wavelength division multiplexing components and related passive optical devices, various fiber-optic transmission system studies, and fiber-optic long haul very high-speed data transport systems. His current interests are in optical techniques for broad-band local access networks, in particular coherent multichannel techniques, subcarrier multiplexing, and optical amplifiers. Since 1991 , he also works as a part-time professor in the Transmission Systems and Technology subgroup of the Tele-Informatics and Open Systems group at the University of Twente. 